\title{
Investigation of iris segmentation techniques using active contours for non-cooperative iris recognition
}

\author{
Akinola Samuel Akinfende, Agbotiname Lucky Imoize, Simeon Olumide Ajose \\ Department of Electrical and Electronics Engineering, University of Lagos, Nigeria
}

\begin{tabular}{l}
\hline Article Info \\
\hline Article history: \\
Received Sep 24, 2019 \\
Revised Mar 17, 2020 \\
Accepted Apr 3, 2020 \\
\hline Keywords: \\
Active contour \\
Biometrics \\
Circular hough transformation \\
False acceptance \\
False rejections \\
GUI-based model \\
Integro-differential-operator \\
Iris recognition \\
Iris segmentation
\end{tabular}

\begin{abstract}
Iris image segmentation process based on Graphical User Interface (GUI) to accurately localize the iris structure is presented in this paper. The major challenge confronting the precision of an iris recognition model is how to determine the accuracy of the iris segmentation and localization. There are varying parameters that introduce constraints during feature extraction and these greatly affect the matching performance during iris localization. To this end, the Integro-differential operator, which involves the detection of inner and outer regions of the iris, and the Circular Hough Transform, which is capable of detecting the circular boundary from the edge mapping were investigated, and an Active Contour model was evolved. In the evolved model, an emerging curve mapped with the zeros of the data set function is experimentally exploited. To demonstrate the suitability of the model for precise iris recognition, its parameters were compared against other related models. Simulation results show that the model has higher flexibility of substitution of images, and the images could be analyzed more accurately with less False Rejections (FR) and False Acceptance (FA) in comparison with the Integro-differential operator. This implies that images could be analyzed faster using the evolved model, and easily substituted especially in situations where the need to care for numerous eye patients occur.
\end{abstract}

Copyright $@ 2020$ Institute of Advanced Engineering and Science. All rights reserved.

\section{Corresponding Author:}

Agbotiname Lucky Imoize,

Department of Electrical and Electronics Engineering, Faculty of Engineering,

University of Lagos, Akoka - Lagos, Nigeria.

Email: aimoize@unilag.edu.ng

\section{INTRODUCTION}

Biometric technology helps to identify or verify individuals based on their distinct physical attributes. These physical attributes could be fingerprint, iris or style of typing, and unique signature-which gives distinct information about an individual, and these attributes could be used in authenticating the identity of the individual. In recent times, this technology is gaining a ground-breaking popularity and it find applications in identity verification in schools, checking in passengers at the airport, access control in restricted areas like factories or production lines, access to some secured database of financial institutions, and in opthamology, which explores the health care area of the iris, retina and eyes [1, 2]. Interestingly, biometrics have proffered candidate solutions to the ubiquitous problem of forgetting a password, and this is achieved through iris recognition modeling.

The major challenge confronting the performance of an iris recognition model is how accurate the iris segmentation and localization could be. There are varying parameters that introduce constraints during feature extraction and these greatly impact the matching performance of iris recognition, and these could be as a result of other dynamic environmental factors during image acquisition. It is worthy of note that Iris recognition adopts some mathematical methods of pattern recognition, and the principles of biometrics in order to recognize and classify the iris of the human eyes. Many approaches abound for iris recognition and the corresponding iris images classification. To this end, an iris recognition algorithm gives more information 
and simplifies the complexities of image component analysis. The sequence of biometrics process involves taking the unique feature of specific specimen, standardized segregation of extracted feature, and the conversion of numerical models to biometric layouts [2-4]. A holistic iris recognition system is made up of some major stages as shown in Figure 1 [3], and a brief description of the stages is as follows;

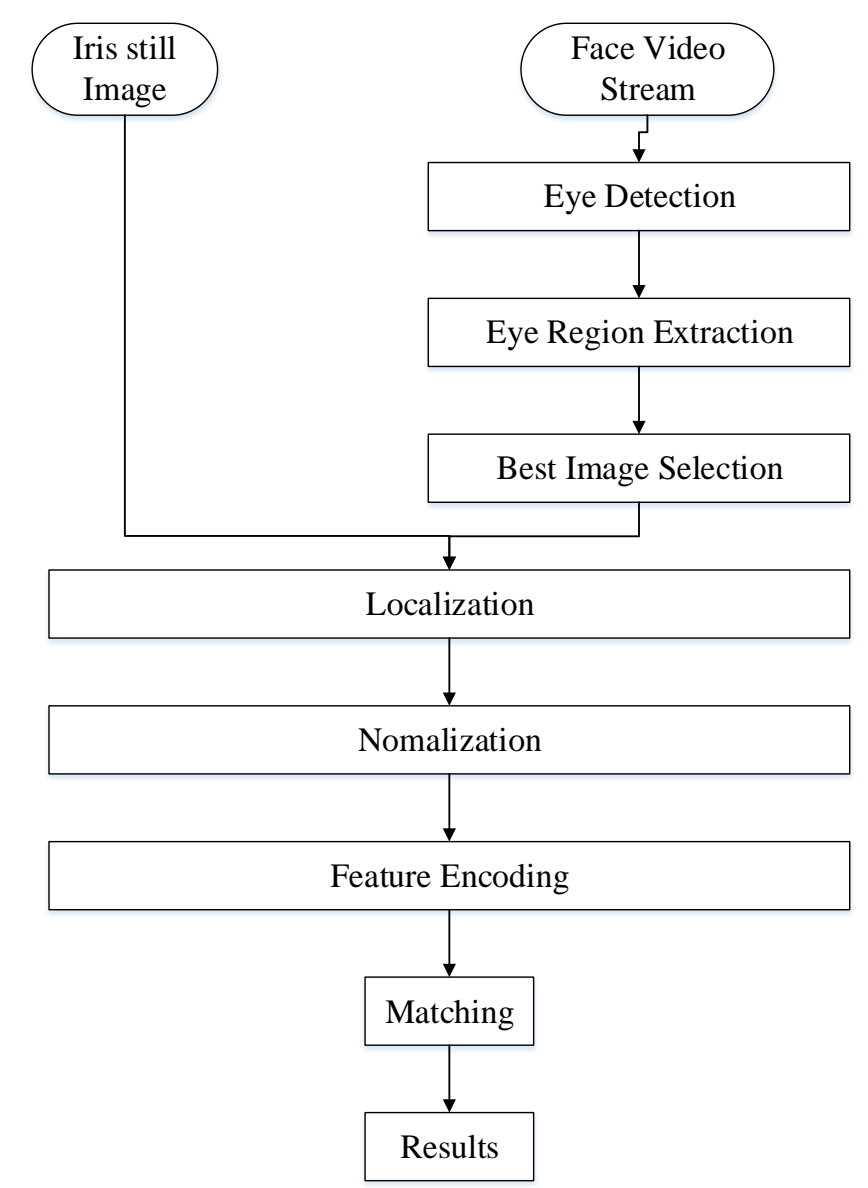

Figure 1. Automated system for iris image processing

a) Image Acquisition: Image acquisition captures the iris images with the incorporation of Infrared illumination. The solution proffered using this method is saving on large storage space requirements for security footage videos, especially in the case of high resolution cameras. Rather than storing large video files, certain camera applications may only need to record video snapshots, which are essentially pictures from a live video stream. The images investigated were obtained using the CCTV camera in the snapshot format.

b) Segmentation: This is a process of locating by isolating the exact structure of the iris from an eye image. This procedure is key to an effective iris recognition model. The gradient-based methods are the most used localization algorithms to locate edges between the pupil, iris and the iris sclera [5].

c) Normalization: This is used to achieve invariance to iris size, position and different degree of iris dilation for matching different iris patterns at later stages of the image processing.

d) Feature Encoding and Matching: This process is used to extract as several discriminating features from the iris and this could results in an iris signature.

Several reports suggest that iris recognition system is a very reliable form of biometric system [3-6]. However, the methodologies and results reported in the open literature are by no means exhaustive. Some of these reports have some research gaps to be filled and a few of these deficiencies have been considered and curbed in this paper, and new contributions have been proposed. Simulations and real time experimentations were used to verify the workability of the proposed model.

Commercial use for iris recognition was proposed in [7,8]. These include the detection of the outer and inner boundaries of the iris using Integro-differential operators, and the Cartesian to polar coordinate 
transforms, which is equivalent to the rectangular representation of the iris zone. Gabor filters were used to extract distinct binary vectors and an exclusive-OR operator was used for the matching scores.

In contrast to the gray images methods, iris recognition using binary mapping of the image edges was reported in [9]. The high processing speed, less storage consumption and simplicity of the hardware were benefits exploited using the edge mapping. The Circular Hough Transform was used for segmentation detecting the circular boundary from the edge mapping of the 256 by 256 pixel eye images. In [9], some advantages of non-cooperative iris recognition were clearly highlighted as:

a) Security: Since no cooperation is required, the operators need not know the site of the image-grabber. Apparently, better results are achieved since the operators are unaware of the security measure and hence cannot bypass it.

b) Customer friendly: The customer's cooperation is not required, several images can be repeatedly captured. The fundamental truth that the customer will not have to undergo these tasks will increase the friendliness of the system.

c) High number of recognition: Non-cooperative recognition systems operate on a larger radius of influence which is usually lesser than $1 \mathrm{~m}$. More time for the recognition process will increase the flexibility and total number of verified or identified persons.

Iris recognition using wavelet transform was presented in [10], 1-D images were acquired from the concentric circles of the iris and calculations were made using its wavelet transform, and comparisons were made by dissimilarity functions. In [8], another method of iris authentication was proposed, which focuses on scalability, tilt and invariant size of the system. The method detects the center of the pupil exploiting a differential transform, which uses the round shape of the eyes for effective localization. The method is suggested to have simple computation in comparison to the Integro-differential operators used in [7].

A simple and efficient iris authentication based on extracting iris features was presented in [11]. The method considered the texture and topology of the iris which was scaled and rotated. The image was pre-processed using the edge detection and a combination of wavelet transform and Gabor filters to effectively locate and match the iris were demonstrated.

In [12], an efficient method similar to [11] was proposed. The iris texture was represented as transient signals which were then processed using wavelet transforms. The points of variation were derived from a set of signals forming distinct features. Moreover, in [13], an optimization of the dimension of the derived vectors to improve processing speed and storage by using Haar wavelet transform was reported. This method was shown to have a significant increase in recognition rate. Without degradation on performance of the system, the iris was presented by 87 bits. An investigation of different methods was reported in [14]. The authors showed that it is possible to know areas that give better performance from a data set of iris images, and these areas were exploited to generate rectangular symbols of the iris.

A fast and efficient algorithm, which is robust against interference was proposed in [15], which uses Point Hough Transform (PHT) on pretreated iris images. This algorithm is an improvement on Daugman algorithm to segment images. In [16-18], algorithms used for the identification stages (localization, segmentation, normalisation, feature extraction and matching) of iris recognition were briefly discussed, noting their respective advantages and disadvantages.

Iris Recognition. The core challenge confronted by a recognition system is to acquire quality image of the iris while it is non-invasive to the user [19]. The iris is a small dark object and the eye is a very sensitive part of the human body, and hence, any proposed system for the human eye examination would require cutting-edge engineering. It should be noted that no matter how sophisticated the device for eye examination could be, acquired images of the iris should be sharp and of reasonable resolution to aid better recognition. It is also crucial to have good illumination to acquire a quality contrast in the interior iris pattern, and the acquired images should be well centered and devoid of random noise [19].

The Iris localization process was clearly reported in [20]. First, it finds the center of the pupil through the gray image; next, it further finds four points at the inner region by the direction of the edge map. The voting mechanism begins from the center of the pupil, and locates inner region of the iris according to the four points earlier chosen. Finally, it finds another four points but this time at the inner region by the direction of the edge map. It repeats the same mechanism for voting from the center of the pupil, and locates the inner region of the iris according to the four points earlier chosen. It should be emphasized that this method of localization has a higher speed and accuracy [21].

The objective of an iris authentication process is to extract, represent and compare the unique identified texture seen on the iris surface. As depicted in Figure 2, the frontal region of the iris is such that it is mapped on a 2-D plane showing clearly the eyelids, pupil, and sclera. Hence, the process of segmentation must distinctively and accurately detect the bands that separate the iris from the other intricate parts of the human body. Furthermore, an approximate shape of the iris actual dimension is derived; the segmentation 
process should detect closures due to eyelids that can confuse the features that have been extracted. An inaccurate iris texture encoding may lead to an error in the segmentation results, and hence affect the recognition accuracy as illustrated in Figure 2 [22].

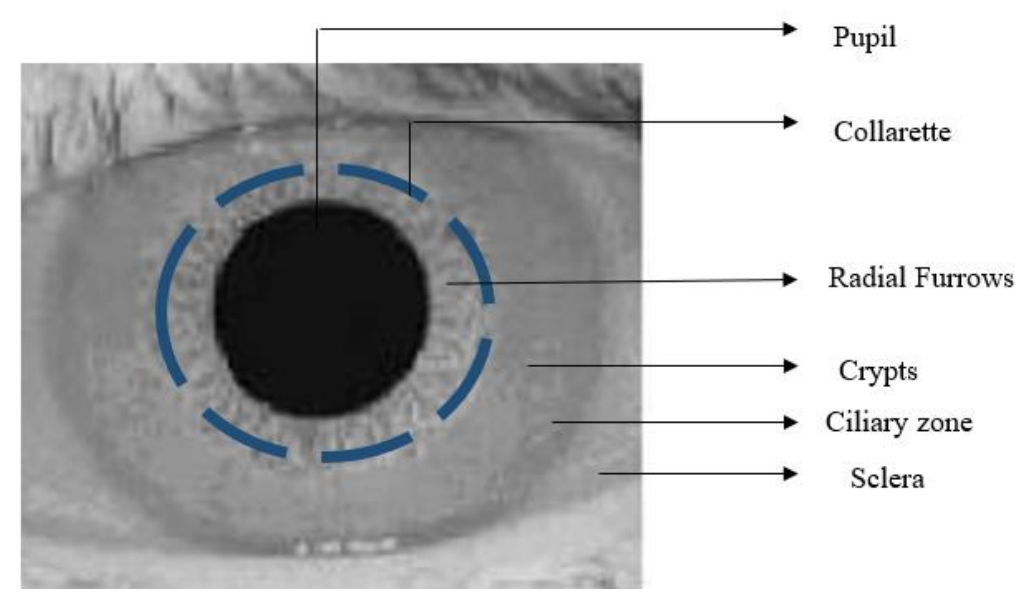

Figure 2. The frontal region of the iris

\section{IRIS MODEL DEVELOPMENT}

The methodology adopted in this paper is quite similar to that reported in [23]. Estimation for the active contours segmentation model by using the data-set based formula for evolution of curves was proposed. The data sets give an inexplicit contour mapping in which an evolving curve is mapped with the zeros of the data set function. The objective of the model is to divide the iris image into two parts which are presumed to represent the object foreground and the background regions.

The detailed flowchat of the Iris recognition model is as shown in Figure 3. The flowchat shows the Iris image acquisition stage fed to the localization and normalization stages, and then enhanced at the image enhancement stage before feature extraction. The extracted features are trained at the train database and tested against predefined metrics or parameters for accurate matching, and a conclusion is reached depending on the matched score. The mathematical formulation is briefly described as follows;

Let $\phi$ be a data set function. Then the CV functional [22] is given in (1)

$$
\mathrm{E}_{C V}\left(c_{1}, c_{2}, \phi\right)=\lambda_{1} \int_{\Omega}\left(f-c_{1}\right)^{2} H(\phi) d x+\lambda_{2} \int_{\Omega}\left(f-c_{2}\right)^{2}(1-H(\phi)) d x+\mu \int_{\Omega}|\nabla H(\phi)| d x
$$

where $\lambda_{1}, \lambda_{2}>0$ and $\mu \geq 0$ are constants. The length parameter $\mu$ can be regarded as a scalar parameter. The detection of smaller objects increases as $\mu$ decreases.

This gives a representation of the segmented image with the variable $c_{1}, c_{2}$ and $H(\phi)$, where $H(\phi)$ denotes the Heaviside function of the data set function $\phi$ given in (2)

$$
H(z)= \begin{cases}1 & \text { if } z \geq 0 \\ 0 & \text { if } z<0\end{cases}
$$

The function $H(\phi)$ identifies foreground and background of the object in the acquired image $f$, while the last term in $(1), \int_{\Omega}|\nabla H(\phi)|$, gives the length of the image boundary. In addition, the scalars $c_{1}$ and $c_{2}$ define the average gray values of the image foreground boundary and the background regions represented by $\phi \geq 0$ and $\phi<0$, respectively.

Hence, the CV model is seen as a two-phase piecewise estimation of the Mumford-Shah (MS) model [24], which can be obtained numerically by making $\alpha$ to tend towards infinity, and two-region segmentation is being forced. To segment an iris image in this context, the CV functional in (1) would need to be minimized with respect to $c_{1}, c_{2}$ and $\phi$. While maintaining $\phi$ constant, the average gray values $c_{1}$ and $c_{2}$ could be deduced in a similar fashion to [22] given in (3) and (4)

$$
c_{1}=\frac{\int_{\Omega} f(x) H(\phi(x)) d x}{\int_{\Omega} H(\phi(x)) d x}
$$




$$
c_{2}=\frac{\int_{\Omega} f(x)(1-H(\phi(x))) d x}{\int_{\Omega}(1-H(\phi(x))) d x}
$$

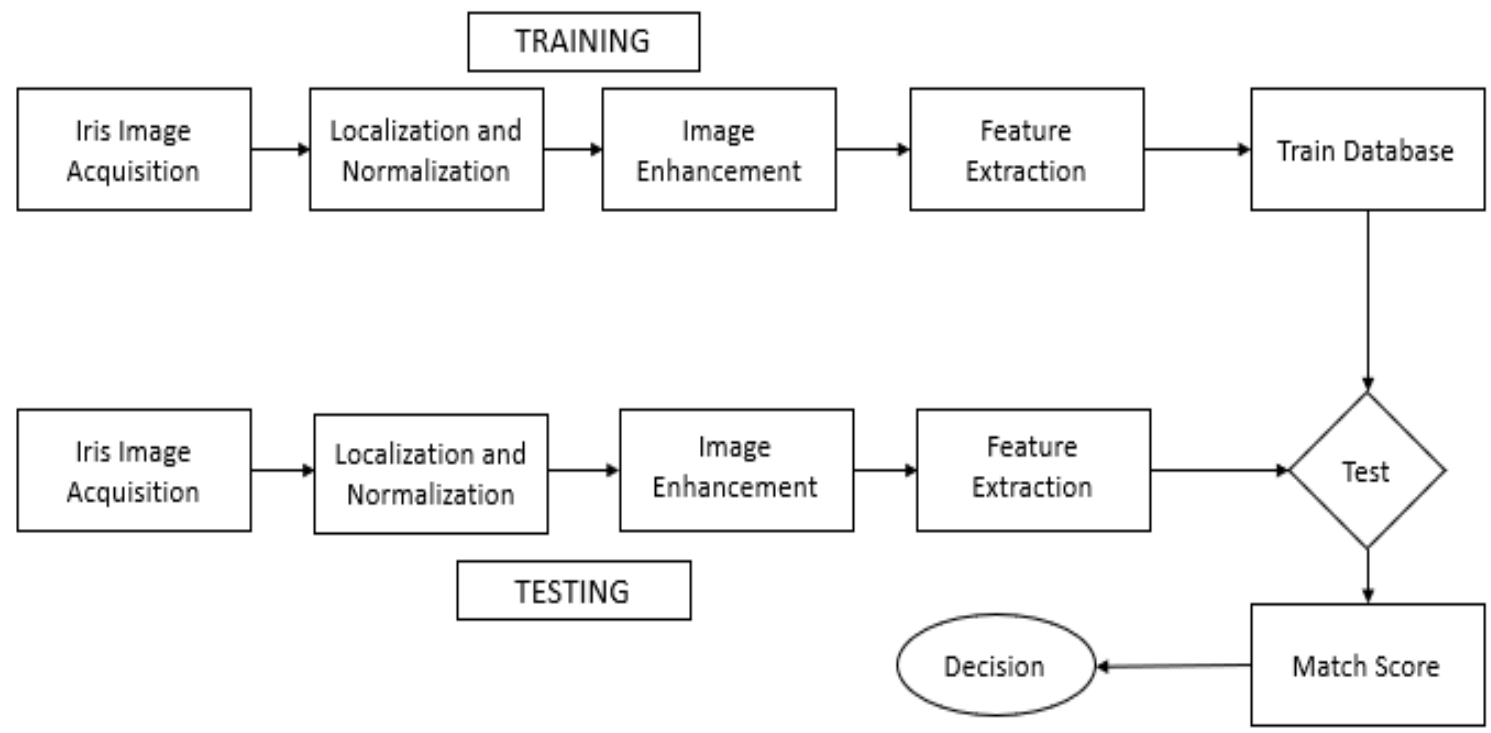

Figure 3. Flowchart of the iris recognition model

\subsection{Model simulation}

The development of the iris image and data needed for the performance evaluations are outlined similar to [25] in the following steps:

a) Defining the mathematical equations that represent the model's parameters

b) Determining the constraints and conditions needed for simulation

c) Writing the Pseudo-codes or algorithms

d) Selecting and organizing the right data structure and functions

e) Writing the main code in steps

f) Debugging the code and making modifications in steps

g) Testing the final program

For the modelling and simulation in this paper, a number of conditions and constraints are listed as follows:

a) Inputs:

1) A 2D Iris image with $100 \times 100$ dimension in jpg format. There are 20 images of the right and left eyes of different individuals.

2) Image Initialization ( $1=$ foreground and $0=$ background $)$

3) Define the number of iterations; 50 iterations were carried out for this study

4) Define the weight of image smoothening. A default of 0.2 was used. A higher value will possibly make the image smoother.

b) Output: The output gives the final segmentation.

\section{RESULTS AND ANALYSIS}

The segmented images using active contours without edges model are expressed in Figure 4(a)-4(e). Five iris images were chosen randomly from a pool of twenty data set. The iris images were first trained and tested on the GUI for the iris segmentation evaluator. The results clearly show the active contour regions for the corresponding segmented images. 


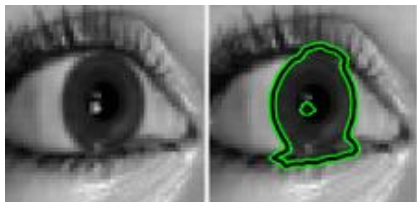

(a)

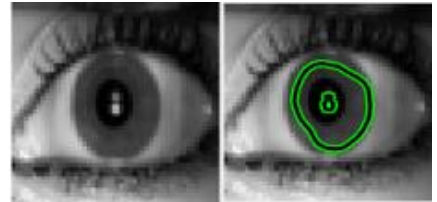

(b)

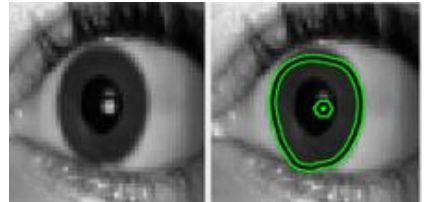

(c)

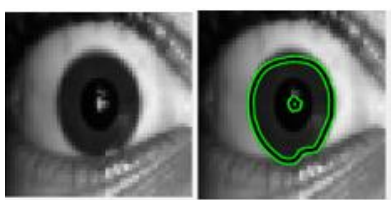

(d)

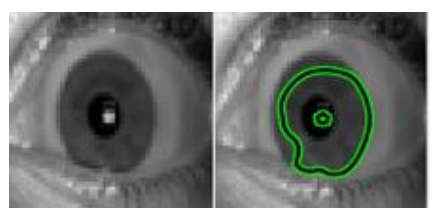

(e)

Figure 4(a)-(e). Segmentation of 5 images showing the active contour in the iris region

\subsection{Results of the GUI-based model}

Figure 5 shows the GUI platform for the iris segmentation evaluation. All tests were carried out using the evaluator as image data are loaded, trained and tested following the procedure in [26]. The test results are well specified in Figure 6 until Figure 16.
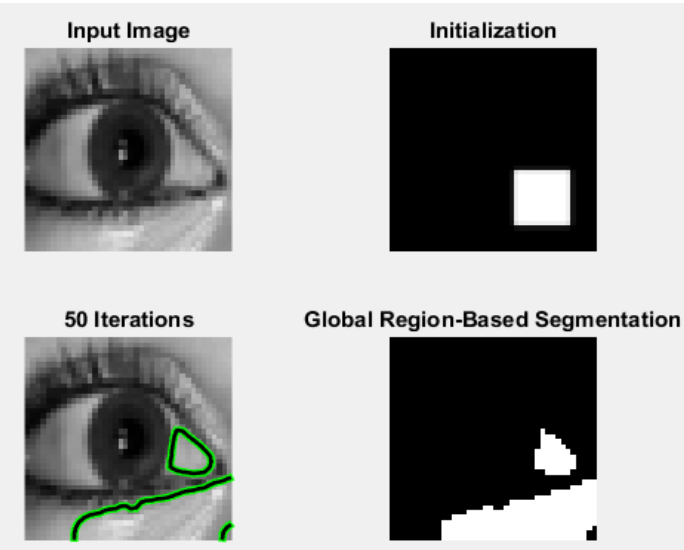

Figure 5. Iris segmentation of image 1 for size 80
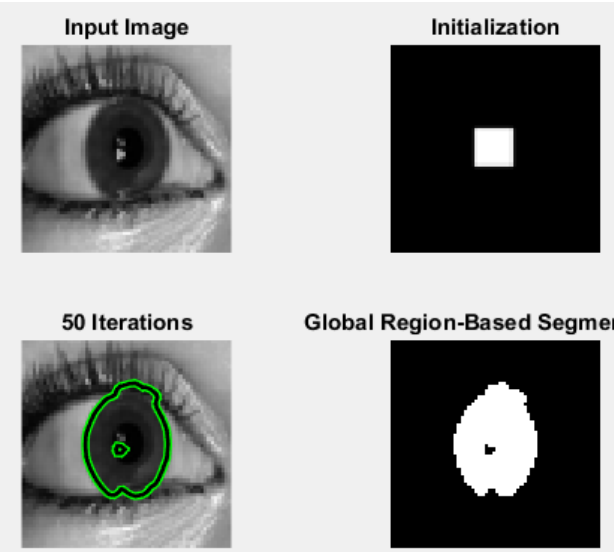

Global Region-Based Segmentation

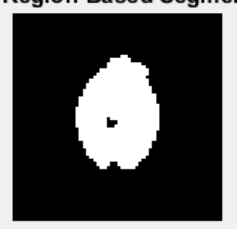

Figure 7. Iris segmentation of image 1 for size 120
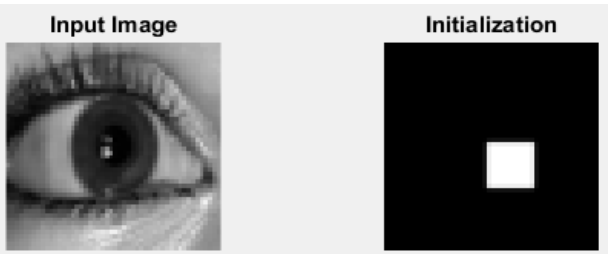

50 Iterations

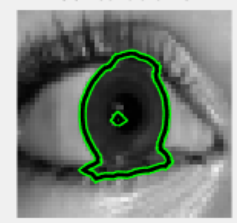

Global Region-Based Segmentation

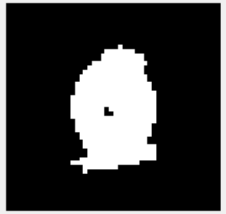

Figure 6. Iris segmentation of image 1 for size 100
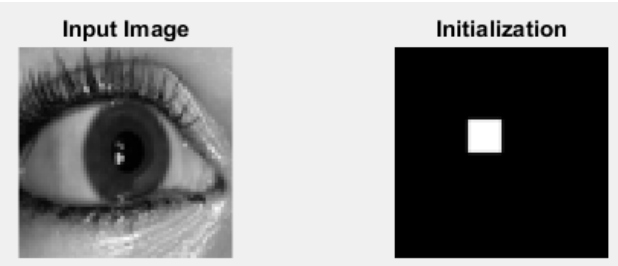

50 Iterations

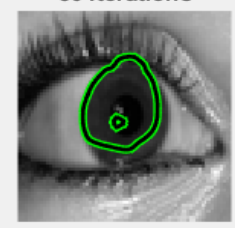

Global Region-Based Segmentation

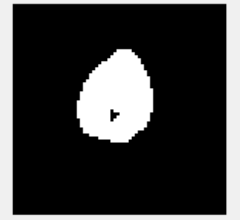

Figure 8. Iris segmentation of image 1 for size 140 

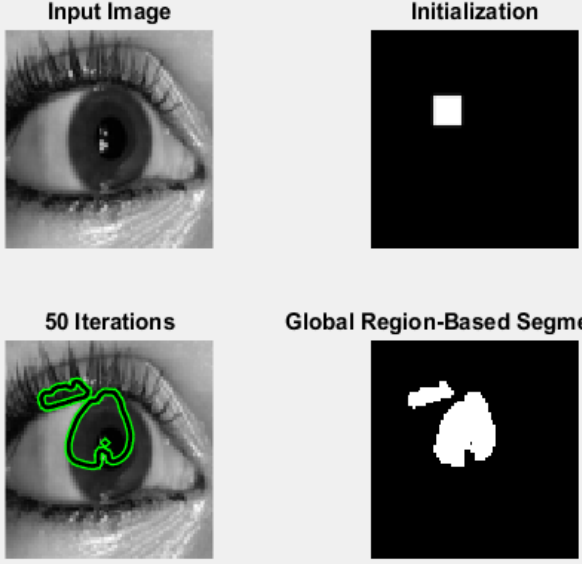

Global Region-Based Segmentation

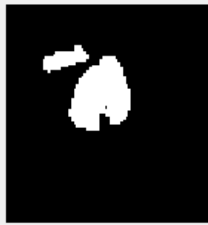

Figure 9. Iris segmentation of image 1 for size 160
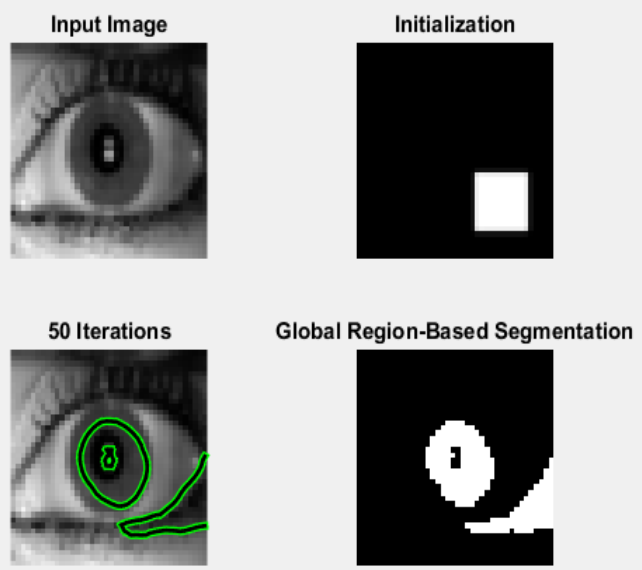

Figure 11. Iris segmentation of image 2 for size 80
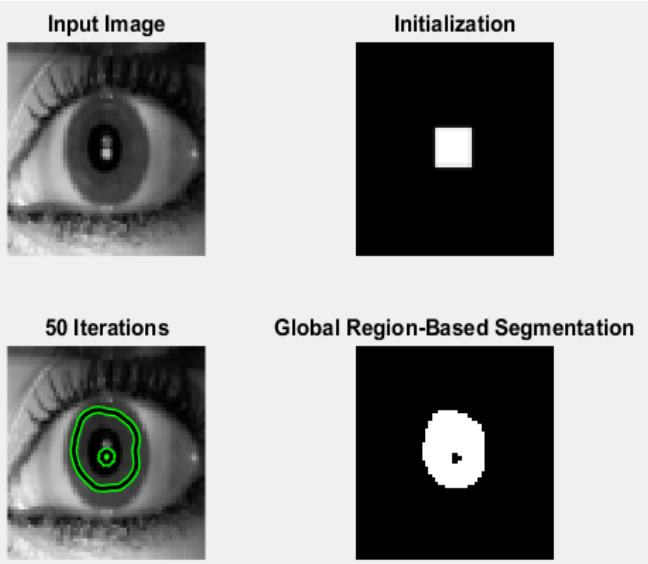

Figure 13. Iris segmentation of image 2 for size 120
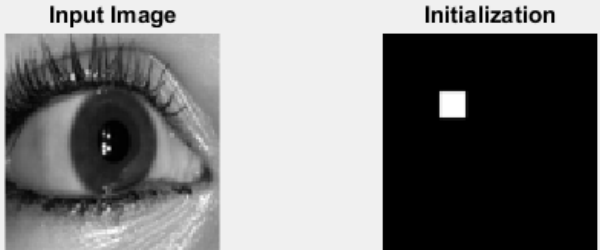

50 Iterations

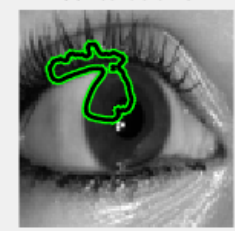

Global Region-Based Segmentation

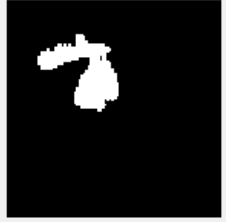

Figure 10. Iris segmentation of image 1 for size 180
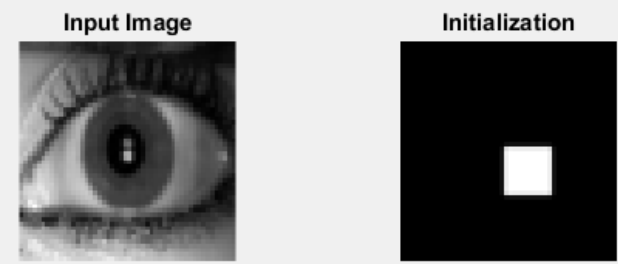

50 Iterations

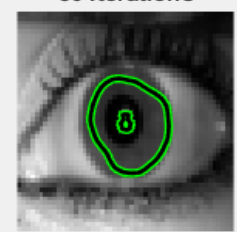

Global Region-Based Segmentation

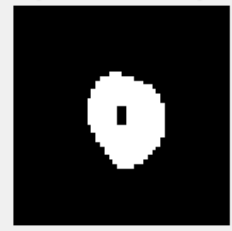

Figure 12. Iris segmentation of image 2 for size 100
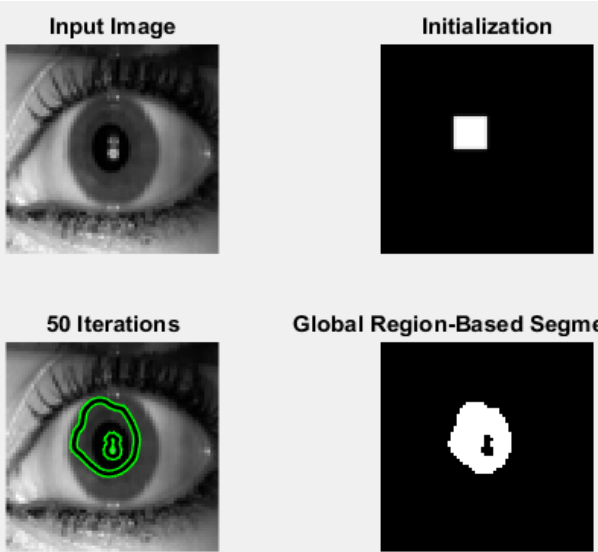

Global Region-Based Segmentation

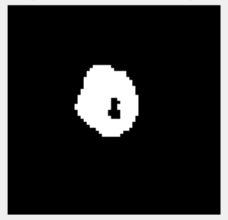

Figure 14. Iris segmentation of image 2 for size 140 


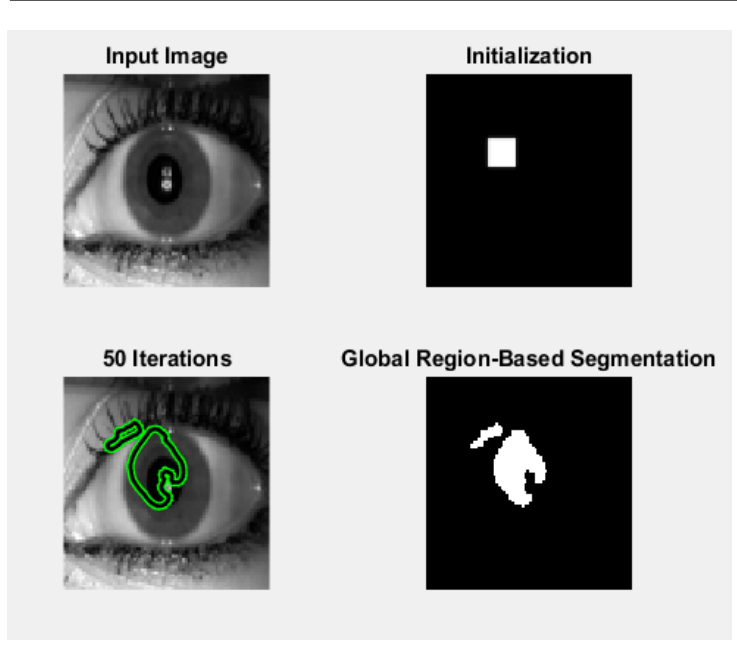

Figure 15. Iris segmentation of image 2 for size 160

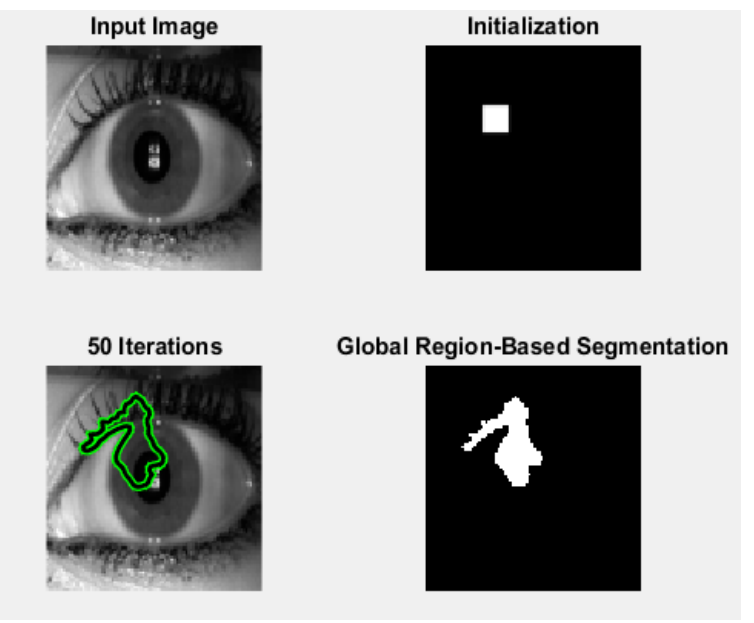

Figure 16. Iris segmentation of image 2 for size 180

\subsection{Comparison with other iris segmentation models}

Table 1 until Table 4 give results of the comparison of the Active Contour without edges model with the Integro-Differential model, and Hough Transform models using False Acceptance (FA), False Rejection (FR), and Recognition Accuracy (RA) as performance metrics. For clarity, the results reported in Tables 3-4 are also illustrated in graphical formats as shown in Figure 17 until Figure 19.

Table 1. FA, FR and RA for total number of iris $=2$

\begin{tabular}{|c|c|c|c|c|c|c|}
\hline & \multicolumn{3}{|c|}{$\begin{array}{c}\text { Total Number of Iris }=2 ; \\
\text { Iris per Individual }=1\end{array}$} & \multicolumn{3}{|c|}{$\begin{array}{l}\text { Total Number of Iris }=2 \\
\text { Iris per Individual }=2\end{array}$} \\
\hline & $\begin{array}{c}\text { Integro- } \\
\text { differential }\end{array}$ & $\begin{array}{l}\text { Hough } \\
\text { Transform }\end{array}$ & $\begin{array}{c}\text { Active } \\
\text { Contour }\end{array}$ & $\begin{array}{c}\text { Integro- } \\
\text { differential }\end{array}$ & $\begin{array}{l}\text { Hough } \\
\text { Transform }\end{array}$ & $\begin{array}{l}\text { Active } \\
\text { Contour }\end{array}$ \\
\hline No of Identified Iris & 2 & 2 & 2 & 4 & 4 & 4 \\
\hline No of Unidentified Iris & 0 & 0 & 0 & 0 & 0 & 0 \\
\hline False Acceptance (\%) & 0 & 0 & 0 & 0 & 0 & 0 \\
\hline \multicolumn{7}{|l|}{ False Rejection (\%) } \\
\hline Recognition Accuracy (\%) & & & & & & \\
\hline Average Recognition time (s) & 2.625 & 67000 & 0.7578 & 5.4531 & 0.2852 & 0.7813 \\
\hline
\end{tabular}

Table 2. FA, FR and RA for total number of iris $=3$ for iris per Individual $=1$ and 2

\begin{tabular}{|c|c|c|c|c|c|c|}
\hline & \multicolumn{3}{|c|}{$\begin{array}{l}\text { Total Number of Iris }=3 \\
\text { Iris per Individual }=1\end{array}$} & \multicolumn{3}{|c|}{$\begin{array}{l}\text { Total Number of Iris }=3 \\
\text { Iris per Individual }=2\end{array}$} \\
\hline & $\begin{array}{c}\text { Integro- } \\
\text { differential }\end{array}$ & $\begin{array}{l}\text { Hough } \\
\text { Transform }\end{array}$ & $\begin{array}{l}\text { Active } \\
\text { Contour }\end{array}$ & $\begin{array}{c}\text { Integro- } \\
\text { differential }\end{array}$ & $\begin{array}{c}\text { Hough } \\
\text { Transform }\end{array}$ & $\begin{array}{l}\text { Active } \\
\text { Contour }\end{array}$ \\
\hline No of Identified Iris & 3 & 3 & 3 & 5 & 6 & 6 \\
\hline No of Unidentified Iris & 0 & 0 & 0 & 1 & 0 & 0 \\
\hline False Acceptance (\%) & 0 & 0 & 0 & 10400.00 & 0 & 0 \\
\hline False Rejection (\%) & & & & 5.1883 & & \\
\hline Recognition Accuracy (\%) & & & & -5090.00 & & \\
\hline Average Recognition time (s) & 2.4635 & 0.3125 & 0.8594 & 2.8906 & 0.2917 & 0.8151 \\
\hline
\end{tabular}

Table 3. FA, FR and RA for total number of iris $=4$ for iris per individual $=1$ and 2

\begin{tabular}{|c|c|c|c|c|c|c|}
\hline & \multicolumn{3}{|c|}{$\begin{array}{l}\text { Total Number of Iris = } 4 \\
\text { Iris per Individual }=1\end{array}$} & \multicolumn{3}{|c|}{$\begin{array}{l}\text { Total Number of Iris = } \\
\text { Iris per Individual }=2\end{array}$} \\
\hline & $\begin{array}{c}\text { Integro- } \\
\text { differential }\end{array}$ & $\begin{array}{l}\text { Hough } \\
\text { Transform }\end{array}$ & $\begin{array}{l}\text { Active } \\
\text { Contour }\end{array}$ & $\begin{array}{l}\text { Integro- } \\
\text { differential }\end{array}$ & $\begin{array}{l}\text { Hough } \\
\text { Transform }\end{array}$ & $\begin{array}{l}\text { Active } \\
\text { Contour }\end{array}$ \\
\hline No of Identified Iris & 4 & 4 & 4 & 7 & 7 & 7 \\
\hline No of Unidentified Iris & 0 & 0 & 0 & 1 & 1 & 1 \\
\hline False Acceptance (\%) & 0 & 0 & 0 & 4740.00 & 1.988 & 1.6401 \\
\hline False Rejection (\%) & & & & 3.3148 & 13.9179 & 1.1494 \\
\hline Recognition Accuracy (\%) & & & & -2270.00 & 92.0471 & 98.6052 \\
\hline Average Recognition time (s) & 2.7266 & 0.3359 & 0.8359 & 2.9141 & 0.332 & 0.9531 \\
\hline
\end{tabular}


Table 4. FA, FR and RA for total number of iris $=5$ and 6 for iris per individual $=2$

\begin{tabular}{|c|c|c|c|c|c|c|}
\hline & \multicolumn{3}{|c|}{$\begin{array}{l}\text { Total Number of Iris }=5 \\
\text { Iris per Individual }=2\end{array}$} & \multicolumn{3}{|c|}{$\begin{array}{l}\text { Total Number of Iris }=6 \\
\text { Iris per Individual }=2\end{array}$} \\
\hline & $\begin{array}{l}\text { Integro- } \\
\text { differential }\end{array}$ & $\begin{array}{l}\text { Hough } \\
\text { Transform }\end{array}$ & $\begin{array}{l}\text { Active } \\
\text { Contour }\end{array}$ & $\begin{array}{l}\text { Integro- } \\
\text { differential }\end{array}$ & $\begin{array}{l}\text { Hough } \\
\text { Transform }\end{array}$ & $\begin{array}{l}\text { Active } \\
\text { Contour }\end{array}$ \\
\hline No of Identified Iris & 8 & 9 & 9 & 9 & 10 & 10 \\
\hline No of Unidentified Iris & 2 & 1 & 1 & 3 & 2 & 2 \\
\hline False Acceptance $(\%)$ & 7030.00 & 5.6088 & 110.2292 & 11600.00 & 6.6579 & 183.4021 \\
\hline False Rejection (\%) & 1.4066 & 50.4821 & 9.9305 & 2.3245 & 16.645 & 4.5872 \\
\hline Recognition Accuracy (\%) & -3420.00 & 71.9545 & 39.9202 & -5710.00 & 88.3485 & 6.0054 \\
\hline Average Recognition time (s) & 3.0813 & 0.3531 & 0.9641 & 2.9883 & 0.3307 & 0.9805 \\
\hline
\end{tabular}

Total Number of Iris $=4$; Iris per Individual $=2$

-Integro-differential $\longrightarrow$ Hough Transform $\longrightarrow$ Active Contour

$$
\begin{aligned}
& \text { No of Identified Iris } \\
& 6000 \\
& 4000 \\
& 2000
\end{aligned}
$$

Recognition Accuracy

$(\%)$

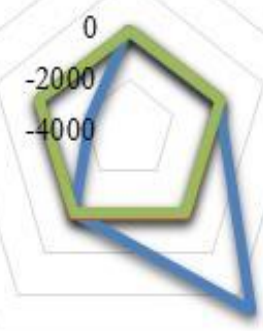

No of Unidentified Iris

False Rejection Rate

False Acceptance Rate

(\%)

$(\%)$

Figure 17. Total number of iris $=4$; iris per individual $=2$

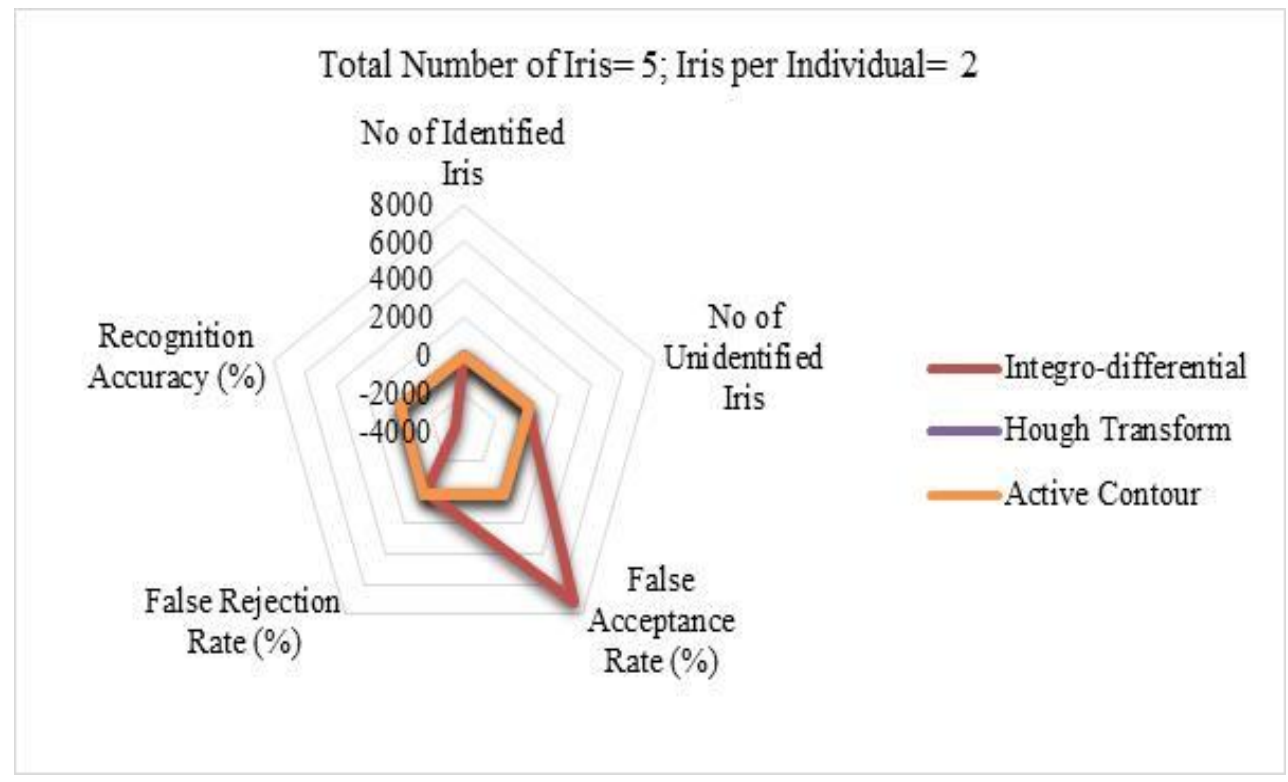

Figure 18. Total number of iris $=5$; iris per individual $=2$ 


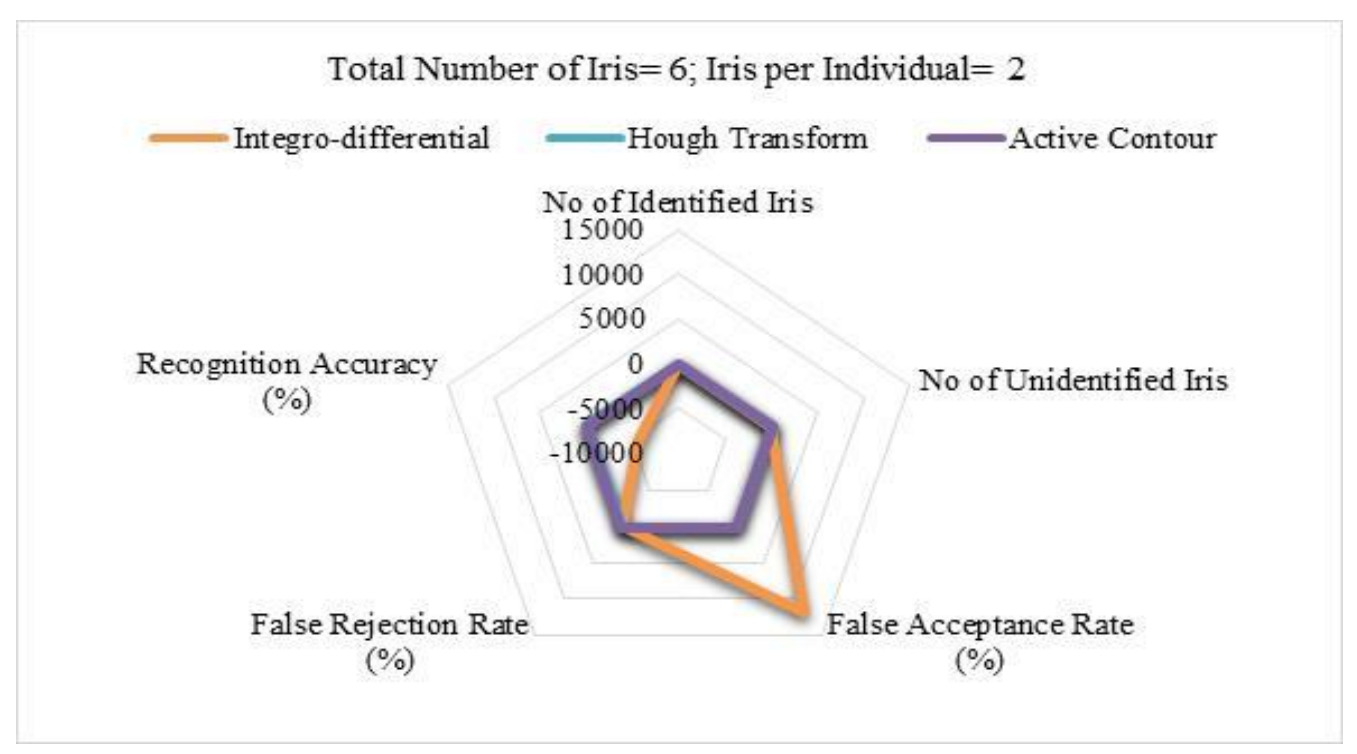

Figure 19. Total number of iris $=6$; iris per individual $=2$

\subsection{Discussion of results}

The results give useful information on the total number of iris trained, number of iris detected after the test was carried out, and the False Acceptance and False Rejection for each test scenario. Figure 5 until Fiure 16 give the results evaluated for active contour after 50 iterations on the basis of the Global Region based segmentation. At lower dimension of image size of 40 to 60 , the image is too blurred and hence, affects the precision for effective iris localization and segmentation processes. Results revealed among other findings that segmentation process becomes effective at an enhancement size of 80 and above.

Furthermore, the results show that at image size 80,140,160,180 and 200 as shown in Figure 6 until Figure 16, the contour just lies outside of the iris towards the sclera region and the eyelids. This shows that for effective iris localization and precise segmentation to be achieved, the image enhancement is clearly between 100 and 120. It should be noted that this process was carried out to attain the most suitable dimensionality for best contour. The 100 by 100 dimension was hitherto used for the entire training and test phases of the investigation. The performances of the Active Contour in relation to the integro-differential operator, and the Hough Transform are presented in Table 1 until Table 4 . These tables appear to present the results using varying number of iris and iris per individual, and these could be related to the results reported by [27-28].

More specifically, it can be seen that at low number of iris images, and the number of individual iris analyzed, the three models performed satisfactorily as depicted in Table 1 until Table 4. However, as the total number of iris images analyzed increases, which suggests a high inflow of eye patients, the Active contours model significantly outperformed the integro-differential model with less False Acceptance and False Rejection as again shown in Tables 1 until Table 4. Finally, Figure 17 until Figure 19 give the graphical representations of the high FA and FR of the Integro differential model as compared to the significantly low FA and FR of the Active contour and Circular Hough Transform.

\section{CONCLUSION}

This paper was focused on an iris image segmentation process that is based on GUI using Active Contours to accurately localize the iris structure for non-cooperative biometric recognition. The strength of the model was observed relative to other well known models to validate improvements in the precision of iris recognition. The proposed model gives the freedom to vary the dimensions of the iris image for greater accuracy of iris detection. Results showed that at image enhancement size of 100 to 120 , the iris localization precision is most suitable for effective segmentation. This was achieved by ensuring that the evolution process was carried out first on a low-pixel image, and an approximate band of 100 to 120 was determined. Results also show that the model has high flexibility of substitution of images, as images could be analyzed more accurately with less False Rejections and False Acceptance as compared to the integro-differential operator model. This implies that images could be analyzed and easily substituted even at increased eye patients' traffic. This study has underscored the importance of an effective segmentation process in iris recognition systems. Most iris systems have explored the integro-differential operator to isolate the iris. The technique implemented in this paper is the Active Contour 
without edges for iris segmentation. The Active Contour model can detect iris images whose regions are not properly identified by its differential, and this makes it best suited for non-cooperative iris recognition. Future work would focus on more efficient techniques for localizing the iris structure, and the results obtained using the Active Contour model without edges would be compared with the performances of its variant models such as the Geodesic Active Contour (GAC) to further test its performance characteristics.

\section{REFERENCES}

[1] M. F. Ab Jabal, et al., "Implementation of Geodesic Active Contour Approach to Localize Anomaly Marker on Iris Surface," Journal of computer science, vol. 12, no. 11, pp. 564-571, 2017.

[2] F. Jan, et al., "Iris localization in frontal eye images for less constrained iris recognition systems," Digital Signal Processing, vol. 22, no. 6, pp. 971-986, 2012.

[3] Y. Lee, et al., "An Automated Video-Based System for Iris Recognition," International Conference on Biometrics: Advances in Biometrics, pp. 1160-1169, 2009.

[4] G. Savithiri and A. Murugan, "Performance Analysis on Half Iris Feature Extraction using GW, LBP and HOG," International Journal of Computer Applications, vol. 22, no. 2, pp. 27-32, May 2011.

[5] E. O. Omidiora, et al., "Iris Recognition Systems: Technical Overview," International Journal of Research in Engineering \& Technology, vol. 3, no. 6, pp. 63-72, 2015.

[6] N. Jagadeesh and C. M. Patil, "Iris recognition system development using MATLAB," 2017 International Conference on Computing Methodologies and Communication (ICCMC), Erode, India, pp. 348-353, 2017.

[7] W. S. Chen, et al., "Personal identification technique based on human iris recognition with wavelet transform," Proceedings IEEE International Conference on Acoustics, Speech, and Signal Processing, vol. 2, pp. 949- 952, 2005.

[8] V. G. Garagad and N. C. Iyer, "A Novel Technique of Iris Identification for Biometric Systems," International Conference on Advances in Computing, Communications and Informatics (ICACCI), pp. 973-978, 2014.

[9] N. Sudha, et al., "Iris recognition on edge maps," IET Computer Vision, vol. 3, no. 1, pp. 1-7, 2009.

[10] W. W. Boles and B. Boashash, "A human identification technique using images of the iris and wavelet transform," IEEE Transactions on Signal Processing, vol. 46, no. 4, pp. 1185-1188, 1998.

[11] M. Nabti and A. Bouridane, "An effective and fast iris recognition system based on a combined multiscale feature extraction technique," Pattern Recognition, vol. 41, no. 3, pp. 868-879, 2008.

[12] L. Ma, et al., "Efficient Iris Recognition by Characterizing Key Local Variations," IEEE Transactions on Image Processing, vol. 13, no. 6, pp 739-750, 2004.

[13] S. Lim, et al., "Efficient Iris Recognition through Improvement of Feature Vector and Classifier," ETRI Journal, vol. 23, no. 2 pp. 61-70, 2001.

[14] J. M. H. Ali and A. E. Hassanien, "An Iris Recognition System to Enhance E-security Environment Based on Wavelet Theory," Advanced Modeling and Optimization, vol. 5, no. 2, pp. 93-104, 2003.

[15] Q. Guo and J. Zheng, "An Iris Recognition Algorithm for Identity Authentication," in 2018 International Conference on Intelligent Transportation, Big Data \& Smart City, Xiamen, China, pp. 621-624, 2018.

[16] A. Bansal, et al., "Trends in Iris Recognition Algorithms," in 2010 Fourth Asia International Conference on Mathematical/Analytical Modelling and Computer Simulation, Bornea, pp. 337-340, 2010.

[17] S. S. Harakannanavar and V. I. Puranikmath, "Comparative Survey of Iris Recognition," in 2017 International Conference on Electrical, Electronics, Communication, Computer, and Optimization Techniques (ICEECCOT), Mysuru, pp. 280-283, 2017.

[18] S. Patil, et al., "A Survey on Iris Recognition System," in International Conference on Electrical, Electronics, and Optimization Techniques, Chennai, pp. 2207-2210, 2016.

[19] L. Zhonghua and L. Bibo, "Iris Recognition Method Based on the Coefficients of Morlet Wavelet Transform," International Conference on Intelligent Computation Technology and Automation, pp. 576-580, 2010.

[20] J. G. Daugman, "High Confidence Visual Recognition of Persons by a Test of Statistical Independence," IEEE Transactions on Pattern Analysis and Machine Intelligence, vol. 15, no. 11, pp. 1148-1161, Nov 1993.

[21] Z. Lin and B. Lu, "Iris recognition method based on the imaginary coefficients of Morlet wavelet transform," Seventh International Conference on Fuzzy Systems and Knowledge Discovery, pp. 573-577, 2010.

[22] T. F. Chan and L. A. Vese, "Active Contours Without Edges," IEEE Transactions on Image Processing, vol. 10, no. 2, pp. 266-277, 2001.

[23] M. A. M. Abdullah, et al., "Fast and Accurate Pupil Isolation Based on Morphology and Active Contour," International Journal of Information and Electronics Engineering, vol. 4, no. 6, pp. 418-422, 2014.

[24] A. Tsai, et al., "Curve evolution implementation of the Mumford-Shah functional for image segmentation, denoising, interpolation, and magnification," IEEE Transactions on Image Processing, vol. 10, no. 8, pp. 1169-1186, 2001.

[25] A. L. Imoize, et al., "Software Intrusion Detection Evaluation System: A Cost-Based Evaluation of Intrusion Detection Capability," Communications and Network, vol. 10, no. 4, pp. 211-229, 2018.

[26] A. L. Imoize and A. E. Babajide, "Development of an Infrared-Based Sensor for Finger Movement Detection," Journal of Biomedical Engineering and Medical Imaging, vol. 6, no. 4, pp. 29-44, 2019.

[27] S. Umer, et al., "Person identification using fusion of iris and periocular deep features," Neural Networks, vol. 122, pp.407-419, 2020.

[28] S. Shah and A. Ross, "Iris Segmentation Using Geodesic Active Contours," IEEE Transactions on Information Forensics and Security, vol. 4, no. 4, pp. 824-836, 2009. 


\section{BIOGRAPHIES OF AUTHORS}
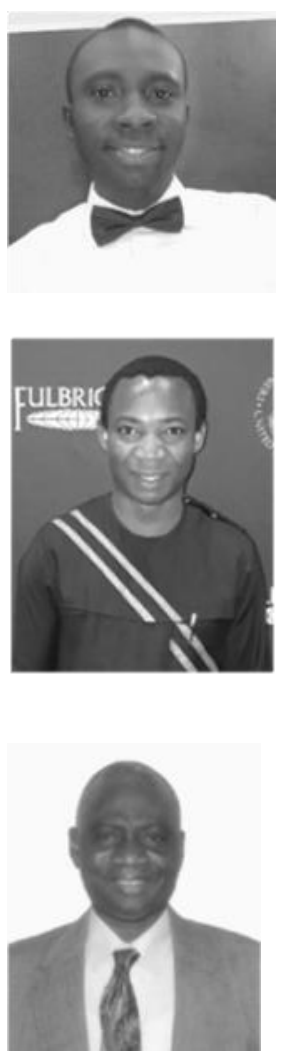

Akinola Samuel Akinfende received his B.Tech (Hons) in Electrical and Electronics Engineering from Ladoke Akintola University of Technology, Ogbomoso, Nigeria, in 2011. He completed M.Sc degree in Electrical and Electronics Engineering with specialization in Communications Engineering at the University of Lagos, Nigeria. He was elected as a Corporate Member of the Nigerian Society of Engineers (NSE), and he is a registered Engineer with the Council for the Regulation of Engineering in Nigeria (COREN). He is a Student Member of IEEE. His research interests are in pattern recognition, image processing, and wireless communication systems.

Agbotiname Lucky Imoize is a Lecturer in the Department of Electrical and Electronics Engineering, University of Lagos, Nigeria. He is a Fulbright Visiting Research Scholar with the Wireless at Virginia Tech Labs, Bradley Department of Electrical and Computer Engineering, Virginia Polytechnic Institute and State University, Virginia, USA. He received his BEng (Hons) in Electrical and Electronics Engineering from Ambrose Alli University, Ekpoma, Nigeria, in 2008, and MSc in Electrical and Electronics Engineering with specialisation in Communications Engineering from University of Lagos, Nigeria, in 2012. He was elected as a Corporate Member of the Nigerian Society of Engineers, and he is a registered Engineer with the Council for the Regulation of Engineering in Nigeria (COREN). His research interests are in the areas of energyefficient wireless communication systems, multi-channel communications, and signal processing.

Simeon Olumide Ajose is a Professor of Electronics and Computer Engineering in Lagos State University. He was the Dean of Engineering from 1998 to 2002. He holds a BSc (Hons) in Electrical Engineering from the University of Lagos, Nigeria (1971), MSc and PhD from the University of London King's College in 1974 and 1976, respectively. He became a Fellow of the Nigerian Society of Engineers (FNSE) in 1989 and was featured in the first issue of International Who's Who in Engineering. His research interests are in the areas of microwave networks, digital signal processing and communication technology. 